\title{
Produção de um planner para assistência de enfermagem no tratamento diretamente observado
}

\author{
Production of a nursing assistance care planner in treatment directly observed
}

\author{
Claudiane Santana Silveira Amorim¹, Fernanda Cruz de Oliveira², Vaneska Tainá Pinto \\ Barbosa $^{3}$, Mônica de Cássia Pinheiro Costa ${ }^{4}$, Sávio Felipe Dias Santos ${ }^{5}$, Maria do Pérpetuo \\ Socorro Dionizio Carvalho da Silva ${ }^{6}$
}

\section{Resumo}

Objetivos: Relatar a experiência de acadêmicos de enfermagem na construção de uma tecnologia para assistência de enfermagem ao usuário com Tuberculose e em Tratamento Diretamente Observado. Método: Relato de experiência, do tipo descritivo, de abordagem qualitativa, vivenciado por acadêmicos de enfermagem do $5^{\circ}$ ano (10ํㅗ semestre), da Universidade do Estado do Pará, durante o estágio supervisionado de Enfermagem em Saúde Coletiva dentro de uma Unidade Básica localizada na região metropolitana de Belém do Pará, acerca de uma proposta inovadora para assistência de enfermagem no TDO, no período de fevereiro a março de 2019. Resultados: O processo de elaboração da proposta inovadora - Planner - seguiu através das fases de definição e desenvolvimento. As fases de definição ocorreram em três fases especificas, denominadas: planejamento, analise e revisão. Após a produção do Planner, o grupo pode observar que este é fundamental para criar mudanças dentro de um cenário que apresenta dificuldades e obstáculos, sejam eles estruturais ou funcionais, uma vez que dinamiza o serviço e a compreensão dos usuários, favorecendo a consulta e adequando-a aos limites de cada usuário. Conclusão: As ferramentas administrativas são essenciais para melhorar a qualidade do acesso, de vida e do reconhecimento dos usuários enquanto protagonistas da sua saúde.

Palavras-Chave: Tuberculose; Tratamento Diretamente Observado; Inovação Tecnológica.

\section{Abstract}

Objectives: To report the experience of nursing academics in the construction of a technology for nursing care to the user with Tuberculosis and in Directly Observed Treatment. Method: This is a descriptive experience, of qualitative approach experienced by nursing students of the 5th year (10th semester), of the University of the State of Pará, during the supervised internship of Nursing in Public Health within a Basic Unit located in the metropolitan area of Belém do Pará, about an innovative proposal for nursing care at the TDO, from February to March, 2019. Results: The process of preparing the innovative proposal - Planner - followed through the phases of definition and development. The definition phases occurred in three specific phases, called: planning, analysis and revision. After the creation of Planner, the group can observe that it is fundamental to create changes

\footnotetext{
1 Graduanda em Enfermagem. Universidade do Estado do Pará. Email: claudianeuepa@gmail.com 2 Graduanda em Enfermagem. Universidade do Estado do Pará. Email: nadacruzoli9@email.com 3 Graduanda em Enfermagem. Universidade do Estado do Pará. Email: vaneska.p100@gmail.com 4 Graduanda em Enfermagem. Universidade do Estado do Pará. Email: monica.cst@hotmail.com 5 Graduando em Enfermagem. Universidade do Estado do Pará. Email: saaviofelipe@gmail.com 6 Mestre em Enfermagem. Universidade do Estado do Pará. Email: sdioniz@hotmail.com

Correspondência: Cidade Universitária Prof. José da Silveira Neto - Campus Profissional II - Complexo Saúde - Rua Augusto Corrêa, 01 - Guamá - Belém, PA, Brasil. CEP: 66075-110.
} 
within a scenario that presents difficulties and obstacles, be they structural or functional, since it dynamizes the service and the understanding of the users, favoring the consultation and adjusting a to the limits of each user. Conclusion: That administrative tools are essential to improve the quality of access, life and recognition of users as protagonists of their health.

Keywords: Tuberculosis; Directly Observed Treatment; Tecnologic innovation.

\section{Introdução}

A Tuberculose (TB) é uma doença infecto contagiosa de agente único, o Mycobacterium tuberculosis, superando o número de mortes pelo Vírus da Imunodeficiência Humana (HIV). Em 2017, 10,4 milhões de pessoas adoeceram de TB no mundo, e cerca de 1,3 milhão de pessoas morreram em decorrência da doença ${ }^{1}$.

No Brasil, em 2017 foram notificados 69.569 casos novos da doença. Nesse mesmo ano, o coeficiente de incidência foi igual a 33,5 casos/100 mil habitantes, corroborando com os estudos que demonstram que o país é um dos principais países em casos de TB no mundo ${ }^{1}$. A doença compromete principalmente os pulmões, podendo, entretanto, manifestar-se clinicamente de inúmeras maneiras e em diversos órgãos².

Uma das medidas para combater a TB é por meio da prática correta do Tratamento Diretamente Observado (TDO), em que o usuário está em contato diário com o profissional, criando assim um vínculo eficaz para a continuidade do cuidado. O TDO é um dos pilares da estratégia Directly Observed Treatment Short-Course (DOTS), recomendada internacionalmente, que atua como um importante instrumento para diminuir a resistência aos medicamentos, incentivar a adesão terapêutica e reduzir o abandono do tratamento. No atual panorama do combate à TB, o abandono do tratamento permanece como um dos principais desafios para o sistema de saúde brasileiro ${ }^{3}$, que, a partir de 2000, estendeu suas ações para a atenção primária, visando uma articulação com a Estratégia Saúde da Família (ESF), objetivando expandir o TDO para todo o país ${ }^{4}$.

Nessa perspectiva, a linha de cuidado da pessoa com TB é caracterizada por uma estratégia de reorganização dos serviços de saúde ${ }^{5}$, dessa forma, o profissional que está diretamente ligado ao tratamento do paciente com TB estar em constante capacitação, conhecendo novas abordagens do cuidado, fazendo uso de instrumentos metodológicos facilitadores e efetivadores de cuidado.

A respeito especificamente da assistência de enfermagem, pode-se dizer que se baseia em duas bases de atuação: tecnologia do cuidado, no sentido do saber fazer, e o valor da vida, tendo como princípios a moralidade e ética do seu trabalho, os quais são possíveis graças a formação profissional, 
a produção científica e filosófica e as estratégias políticas ${ }^{6}$.

Nesse cenário, observa-se a crescente a necessidade de se usar as Tecnologias Educacionais (TE), na medida em que vêm sendo planejadas e implementadas no sentido de transformar ou dispor o conhecimento técnico-científico em forma de ferramentas facilitadoras para o cuidado e para difundir informações, e dessa forma qualificar a assistência?

Diante do exposto, o presente relato objetiva apresentar uma proposta inovadora à assistência de enfermagem no TDO, a fim de produzir um instrumento metodológico (especificamente, um Planner) para auxiliar e estimular tanto o profissional de saúde, como também o usuário dos serviços de saúde que fazem tratamento de TB a realizar 0 tratamento corretamente, conforme as recomendações do Ministério da Saúde (MS). A relevância dessa proposta se justifica tendo em vista as estimativas alarmantes do número de pessoas acometidas pela doença, tanto em nível mundial quanto nacional.

Diante de tais problemáticas, foi elaborado o seguinte questionamento para o desenvolvimento desse estudo: como contribuir para uma maior adesão do usuário com TB ao tratamento?

\section{Método}

Trata-se de um relato de experiência, do tipo descritivo, de abordagem qualitativa, vivenciado por acadêmicos de enfermagem do $5^{\circ}$ ano (10ำ semestre), do curso de enfermagem da Universidade do Estado do Pará, durante o estágio supervisionado de Enfermagem em Saúde Coletiva dentro de uma Unidade Básica localizada na região metropolitana de Belém do Pará, acerca de uma proposta inovadora para assistência de enfermagem no TDO, no período de fevereiro a março de 2019.

A escolha do local para realização dessa proposta se deu, pois nela o TDO apresentava dificuldades em ser realizado conforme as recomendações do MS. Por ser uma unidade localizada em região estratégica com bairros periféricos e atendendo também a livre demanda, desempenha papel importante dentro da comunidade, uma vez que é uma estrutura de baixa e média complexidade entre o usuário e o hospital.

A partir da observação da prática do TDO desenvolvida pelo enfermeiro na unidade, produziu-se uma tecnologia inovadora educativa/organizacional com a proposta de gerenciar o cuidado no programa de TB. Nesse sentido, foi produzido um Planner, uma ferramenta administrativa que tem como objetivo organizar e sistematizar as ações diárias da pessoa dentro de um contexto social (trabalho, escola, afazeres de casa, entre outras atividades) a fim de que exista um resultado eficiente diante do programado.

A produção desse instrumento foi pactuada em três etapas: ambientação à unidade; busca nas literaturas atuais, bem 
como recomendações do MS e construção propriamente dita.

Primeiramente, 0 grupo foi apresentado a Unidade e a todos os serviços que a integrava juntamente com 0 direcionamento do docente orientador para observar e destacar as principais fragilidades do serviço, a partir desse momento, pode-se observar que o serviço de TB e Hanseníase eram os que apresentavam mais fragilidades em detrimento dos outros, principalmente no que diz respeito a gestão e gerenciamento dos medicamentos durante o TDO e também sobre o protagonismo do usuário dentro do seu autocuidado diante da TB.

O Planner desenvolvido é constituído de dados sociais e econômicos do paciente, a descrição rápida do uso da ferramenta, a síntese sobre a TB e os tipos de tratamento, assim como os principais efeitos adversos, além disso conta com os meses de tratamento, a fim de que o próprio usuário organize e manifeste suas principais dúvidas e acontecimentos daquele mês específico.

Cabe salientar que a ideia de produzir uma proposta inovadora (planner organizacional) foi o primeiro passo do estudo, seguindo em futuras pesquisas a validação desse instrumento para poder, a partir de resultados positivos, entregar a comunidade.

\section{Resultados}

O processo de elaboração da proposta inovadora seguiu através das fases de definição e desenvolvimento. As fases de definição ocorreram em quatro etapas especificas, quais são: planejamento, analise, revisão e produção da tecnologia inovadora.

Planejamento: ocorreu a partir da análise minuciosa do Aplicativo "Canva", identificando suas funções de editor, criação de design e imagens profissionais para o desenvolvimento do Planner.

Análise: analisou-se todo o conteúdo educativo e design desenvolvido, levando em consideração a finalidade a ser alcançada com o uso da proposta tecnológica inovadora, com objetivo de aperfeiçoar e informatizar o desenvolvimento do Planner.

Revisão: consistiu em observar se as necessidades dos pacientes estão sendo abordadas dentro da tecnologia inovadora e que o mesmo pode vir ser utilizado como uma ferramenta para efetivar o TDO, reduzindo a incidência ao abandono do tratamento.

Produção da tecnologia inovadora: o Plannerfoi produzido, a partir da análise da realidade do local e especificidade do cliente, com o intuito de proporcionar uma Sistematização da Assistência de Enfermagem (SAE) de forma eficiente, a fim de auxiliar no TDO aos pacientes com TB.

O Planner foi intitulado "Organizando meu tratamento", que além de auxiliar no TDO proporciona maior autonomia e responsabilidade dos pacientes para concluir o tratamento até a cura. Quanto a sua estrutura, a página inicial apresenta o título da tecnologia inovadora/educativa junto com a imagem ilustrativa, que foi criada pelo 
próprio aplicativo utilizado para o desenvolvimento do Planner.

A seguir, seguen informações pessoais do paciente como nome, endereço, idade e a unidade de saúde que realiza o tratamento, além de ter o espaço para colocar foto para identificação do usuário. Após, seguem as informações educativas, em tópicos, sobre como usar o Planner, o que é TB, seu tratamento, efeitos adversos e o que é o TDO. Vale ressaltar que todos os conteúdos educativos foram embasados pelo manual de TB do MS e de literaturas atuais.

Ao final de cada tópico abordado seguem as referências utilizadas para 0 desenvolvimento do conteúdo e um espaço para o paciente colocar suas dúvidas quanto ao seu tratamento. Esse espaço foi elaborado com a finalidade de efetivar a relação paciente-enfermeiro, fazendo com que ocorra um feedback positivo durante o tratamento e que todas as dúvidas do paciente possam ser reduzidas, assim efetivando o tratamento até a cura e evitando o abandono do tratamento.

Ao final do Planner, segue 0 planejamento do tratamento de tuberculose, levando em consideração a fase de ataque e de manutenção que dura em torno de 6 meses. Todos os 6 meses vêm com um calendário de segunda a domingo, onde 0 paciente deve marcar as doses administradas em sua casa. Vale ressaltar que o Planner foi produzido para o esquema básico de tratamento para TB.
Após a produção do Planner, o grupo pode observar que a construção de uma TE é fundamental para criar mudanças dentro de um cenário que apresenta dificuldades e obstáculos, sejam eles estruturais ou funcionais, uma vez que a criação de um instrumento organizador, no caso o Planner, dinamiza o serviço e a compreensão dos usuários, favorecendo a consulta e adequando-a aos limites de cada usuário.

O Planner auxiliou o grupo a compreender que 0 profissional de enfermagem tem inúmeras ferramentas no ambiente de trabalho e também fez com que percebêssemos que existe uma construção na academia, no que diz respeito a competências e habilidades administrativas, que nos favorecem compreender melhor os cenários de atuação e como construir um modelo de ação diferenciado do padrão, que auxilie no ambiente de trabalho e na dinâmica entre nós, profissionais de saúde, e o usuário com TB.

Abaixo estão as figuras demonstrativas do resultado final do Planner, organizado em: capa, informações iniciais e adicionais.

A figura 1 (anexos) configura a capa do Planner, contendo o nome e cabeçalho informativo da instituição a qual está vinculado.

A partir da figura 2 (anexos) em diante, pode-se observar a organização do planner em informações, essa figura, especificamente, demostra uma área destinada ao preenchimento dos dados pessoais do usuário. 
A figura 3 (anexos) manifesta a elaboração de uma página especial explicando ao usuário como usar o seu planner, frisando a importância de estar utilizando esse tipo de ferramenta.

As figuras 4, 5, 6 e 7 (anexos) revelam a organização do conteúdo informativo do planner, onde foi abordado a respeito do conceito de TB, tratamento, efeitos adversos e o TDO.

A figura 8 (anexos), refere-se ao conteúdo interno do planner, voltado especialmente para o aprazamento pessoal do usuário, configurando dessa forma, a proposta principal para a criação desse instrumento, possibilitando o protagonismo do usuário frente ao tratamento de TB e proporcionando uma comunicação ativa e efetiva com o profissional de saúde que the acompanha.

\section{Discussão da Experiência}

A partir da observação da realidade foi verificada a necessidade da produção de uma TE para auxiliar o tratamento de TB, pois uma das maiores problemáticas relacionadas ao tratamento é a não adesão e/ou abandono, resultando assim em um fracasso terapêutico e aumento do risco de disseminação da TB na sociedade, principalmente, dentro da periferia, onde foi desenvolvido o estudo. Corroborando assim, com estudo de Freire et. $\mathrm{Al}^{8}$ quando afirma que o combate à TB está diretamente ligado a aceitação dos pacientes ao tratamento. A não adesão resulta no fracasso terapêutico, tornando-se um fator preocupante para saúde pública.

A produduçao da TE (planner) associada ao TDO é um método para organizar o tratamento, influenciar na criação do vinculo profissional e usuário, fortalecer a responsabilização do usuário com relação ao tratamento e incentivar o autocuidado da pessoa com TB. O que é enfatizado pela Organização Mundial da Saúde (OMS) ${ }^{9}$; um sistema de saúde focado nos usuários permite que eles tenham a orientação e o apoio necessários para tomar decisões e gerenciar seus próprios cuidados. Deve-se enfatizar que 0 desenvolvimento de estratégias inovadoras para o cuidado centrado no usuário é incentivado porque a integração e a valorização de indivíduos doentes em seu plano de assistência têm sido associadas a uma melhor adesão ao tratamento e a melhores resultados na qualidade de vida de indivíduos.

Desse modo, a TE caracteriza-se por ser um recurso fundamental para a melhoria da saúde, de modo que há uma real necessidade de desenvolver a atividade de cunho educativo de forma bem planejada, sendo facilitada por algumas condições como: a problematização da realidade, buscando os porquês e a solução desses problemas; a existência dos conhecimentos e competências de profissionais que desejam fazer sua utilização, disponibilidades de recursos, tempo, incentivos, recompensas, participação ativa o compromisso e a 
liderança ${ }^{10}$

Em contrapartida, apesar das literaturas apontarem os diversos benefícios do uso das TE, observa-se que há ainda um número considerável de enfermeiros que desconhecem a importância de estar produzindo, validando e testando as TE antes de serem submetidas e usadas ${ }^{11}$.

Nessa perspectiva, o enfermeiro tem papel fundamental tanto no tratamento da TB quanto na produção de instrumentos que facilitem o cuidado. Nesse sentido, que se ratifica a importância do profissional enfermeiro em se manter atualizado e ter constantes produções cientificas sobre 0 assunto, a fim de difundir experiências, auxiliando outros profissionais a realizarem as mesmas práticas, além da multiplicação de informações que conduzam a população ao desenvolvimento do autocuidado, enfatizando o tripé de promoção, prevenção e reabilitação da saúde ${ }^{13}$.

\section{Conclusão}

Ao final da construção da TE de apoio aos usuários com TB e que estão em TDO, pode-se concluir que as ferramentas administrativas são essenciais para melhorar a qualidade do acesso, de vida e do reconhecimento dos usuários enquanto protagonistas da sua saúde.

O Planner dinamiza as ações dos usuários frente a doença e os coloca em uma posição de destaque, proporcionando assim um agir frente a todo o tratamento, por outro lado, o Planner auxilia também o profissional de saúde, que consegue através do instrumento, identificar as principais reações dos usuários em um mês especifico, assim como também quais serão as melhores condutas para seguir, a partir de uma dúvida ou alguma consequência descrita no Planner.

Além disso, percebeu-se que o tema é relevante para academia uma vez que existem poucos trabalhos que ressaltam as tecnologias como uma forma de um acesso equânime e eficiente dentro dos serviços implantados na Atenção Primária em Saúde.

Nesse sentido, sugere-se que 0 Planner possa ser um instrumento facilitador e transmissor da educação em saúde para pessoas em tratamento de TB, a fim de proporcionar maior comprometimento do paciente ao tratamento, protagonismo, diminuição de dúvidas e relação ativa e efetiva com o profissional de saúde.

Assim também como despertar dos profissionais de saúde o interesse por produzir, validar e utilizar as TE no seu ambiente de trabalho, visto que os instrumentos tecnológicos facilitadores para desenvolver as atividades educacionais, bem como potencializar e qualificar a assistência, são essenciais para alcançar resultados positivos frente a realidades problemáticas.

O presente estudo está desprovido de qualquer conflito de interesse ou interesse pessoal/comercial.

\section{Referências}

1. World Health Organization. Bending the curve: ending TB. Annual report 2018 [Internet]. Geneva: World Health 
Organization; 2018 [cited 2019 May 8]. 72 p. Available in: http://apps.who.int/iris/ handle/10665/254762.

2. Veronesi R, Focaccia R. Tratado de Infectologia. Quarta Edição. Rio de Janeiro: Atheneu; 2010.

3. Cruz MM, Cardoso GCP, Abreu DMF, Decotelli PV, Chrispim PP, Borenstein JS, Santos EM. Adesão ao tratamento diretamente observado da tuberculose sentido atribuído pelos usuários e profissionais de saúde em duas regiões administrativas do município do Rio de Janeiro. Cad Saude Colet. 2012 [citado em 07 mai 2019]; 20(2):217-24. Disponível em: http://www.iesc.ufrj.br/ cadernos/images/csc/2012_2/artigos/csc_v2 0n2_217-224.pdf.

4. Alves RS, Souza KMJ, Oliveira AAV, Palha PF, Nogueira JA, Sá LD. Abandono do tratamento da tuberculose e integralidade da atenção na estratégia saúde da família. Texto contexto - enferm. 2012 [citado em 07 mai 2019]; 21(3):650-7. Disponível em: http://

www.scielo.br/pdf/tce/v21n3/v21n3a21.pdf. 5. Silva KF, Pucci VR, Flores TG, Giaretton DWL, Weiller TH, Concatto MEP, Damaceno AN. Construindo a linha do cuidado do paciente oncológico paliativo em munícipio do sul do Brasil: relato de experiência. Rev. APS. 2018 [citado em 08 mai 2019];

21(3):470-77. Disponível em:

https://repositorio.ufsm.br/bitstream/handle/1 /247/Silva_Kauana_Flores_da.pdf?sequence $=1$ \&isAllowed $=\mathrm{y}$.

6. Salvador PTCO, Oliveira RKM, Costa TD, Santos VEP, Tourinho SFV. Tecnologia e inovação para o cuidado de enfermagem. Rev. Enferm. UERJ. 2012 [citado em 08 mai 2019]; 20(1):111-7. Disponível em: http://www.facenf.uerj.br/v20n1/v20n1a19.pd f.

7. Barbosa EMG, Sousa AAS, Vasconcelos MGF, Carvalho REFL, Oriá MOB, Rodrigues DR. Tecnologias educativas para promoção do (auto) cuidado de mulheres no pós-parto. Rev Bras Enferm [internet]. 2015 [citado em 26 set 2019]; 69(3):582-90. Disponível em: http://dx.doi.org/10.1590/0034-

7167.2016690323i.

8. Freire ILS, Santos FR, Menezes LCC, et al. Adesão dos Idosos às Formas de

Administração do Tratamento da
Tuberculose. Rev Fund Care Online.2019. abr./jun.; 11(3):555-559. Disponível em: http://dx.doi.org/10.9789/2175-5361.2019. v11i3.555-559.

9. Organização Mundial da SaúdeOMS. Implementando a estratégia final para a tuberculose: o essencial. Genebra. OMS, 2015. Disponível em:

http://www.who.int/tb/publications/2015/The Essentials_to_End_TB/en/ 10. Silva DML, Carreiro FA, Mello R. Tecnologias educacionais na assistência de enfermagem em educação em saúde: revisão integrative. Rev Enferm UFPE on line. UFPE. 2017. [citado em 26 set 2019]; 11(supl.2):1044-51. Disponível em: DOI: 10.5205/reuol.10263-91568-1RV.1102sup201721. 11. Elisabeth $\mathrm{T}$. tecnologias em enfermagem: produções e tendências para educação em saúde com a comunidade. Rev. Eletr. Enf. 2010. [citado em 30 set 2019]; 12(suppl 4): 598. Diponível em: http://dx.doi.org/10.5216/ree.v12i4.12470. 12. Quental LLC et al. Práticas educativas com gestantes na atenção primária a saúde. Revista de Enfermagem UFPE on line, Recife. 2017. [citado em 30 set 2019]; 


\section{Anexos}

Figura 1: capa do Planner "Organizando Meu Tratamento"

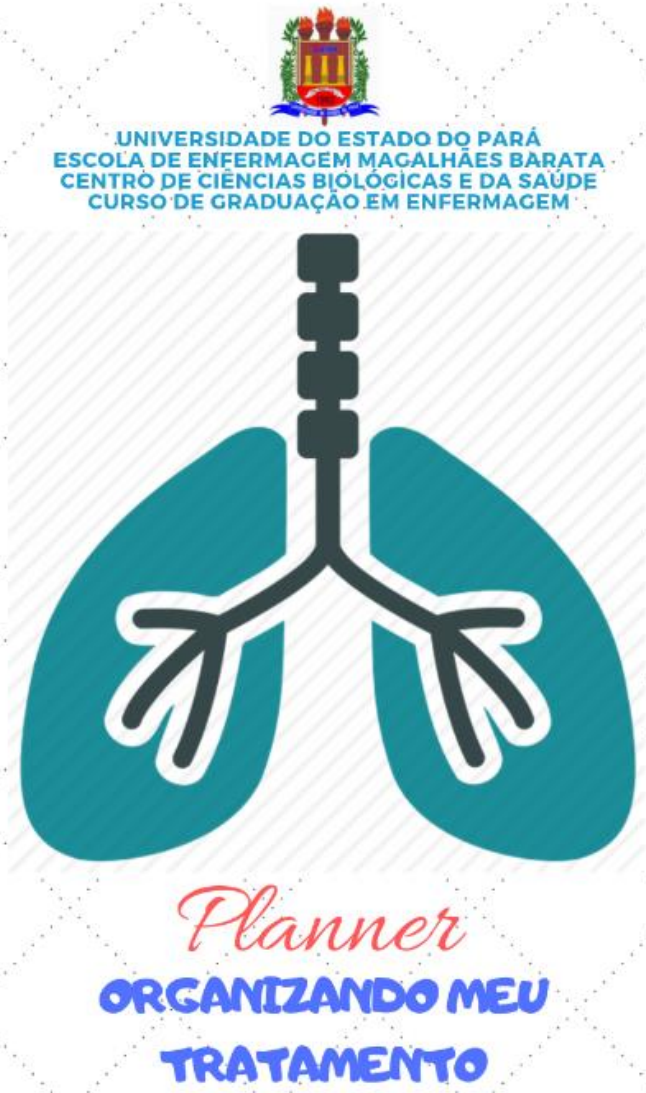

Fonte: acervo pessoal dos autores
Figura 2: Informações iniciais do Planner "Organizando Meu Tratamento"

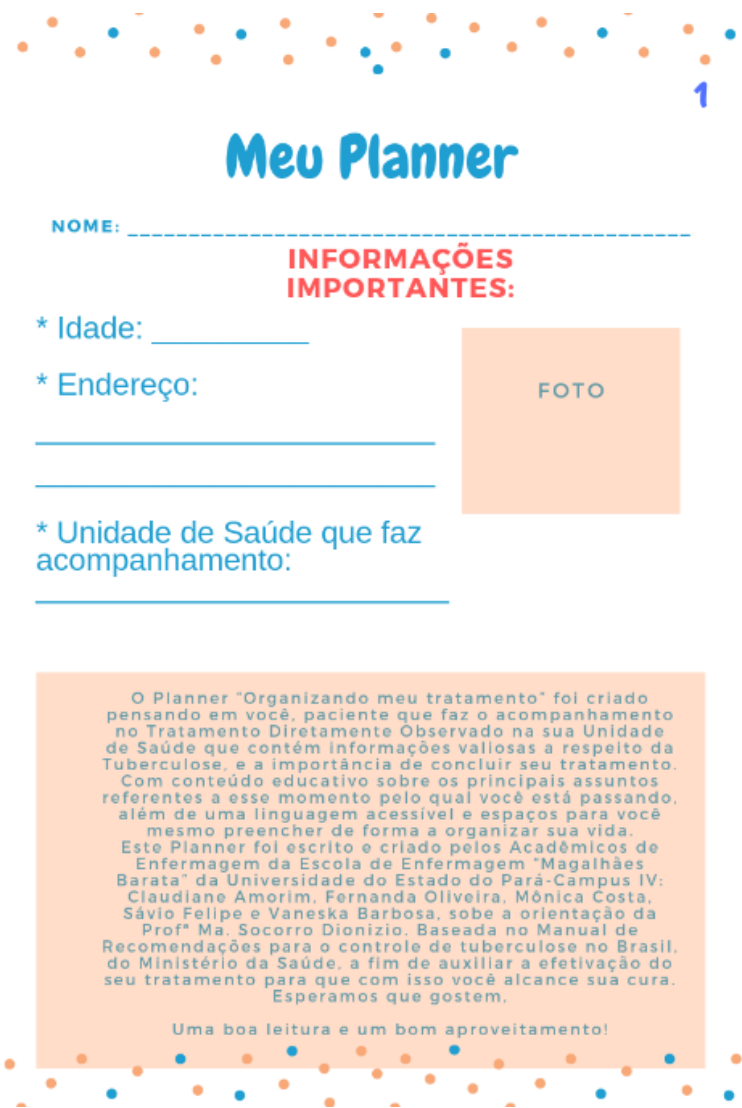

Fonte: acervo pessoal dos autores 
Figura 3: Informações sobre como usar o Planner

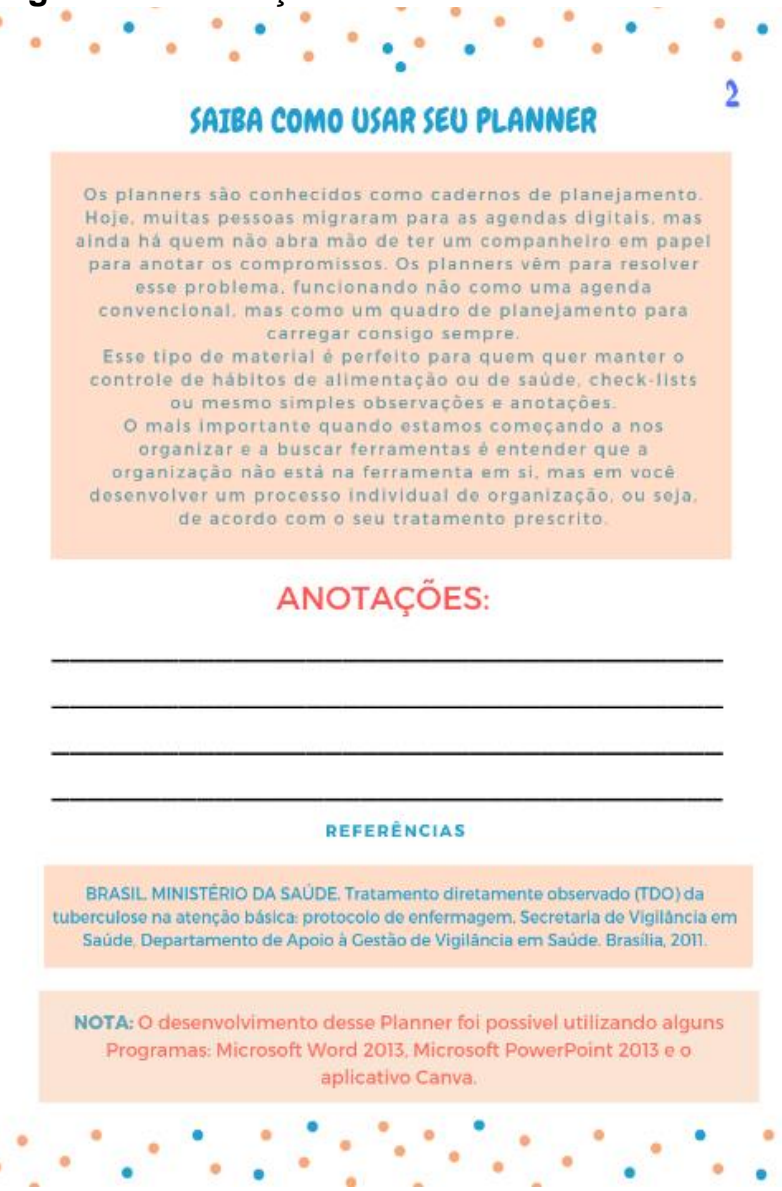

Fonte: acervo pessoal dos autores
Figura 4: Informações adicionais sobre a Tuberculose

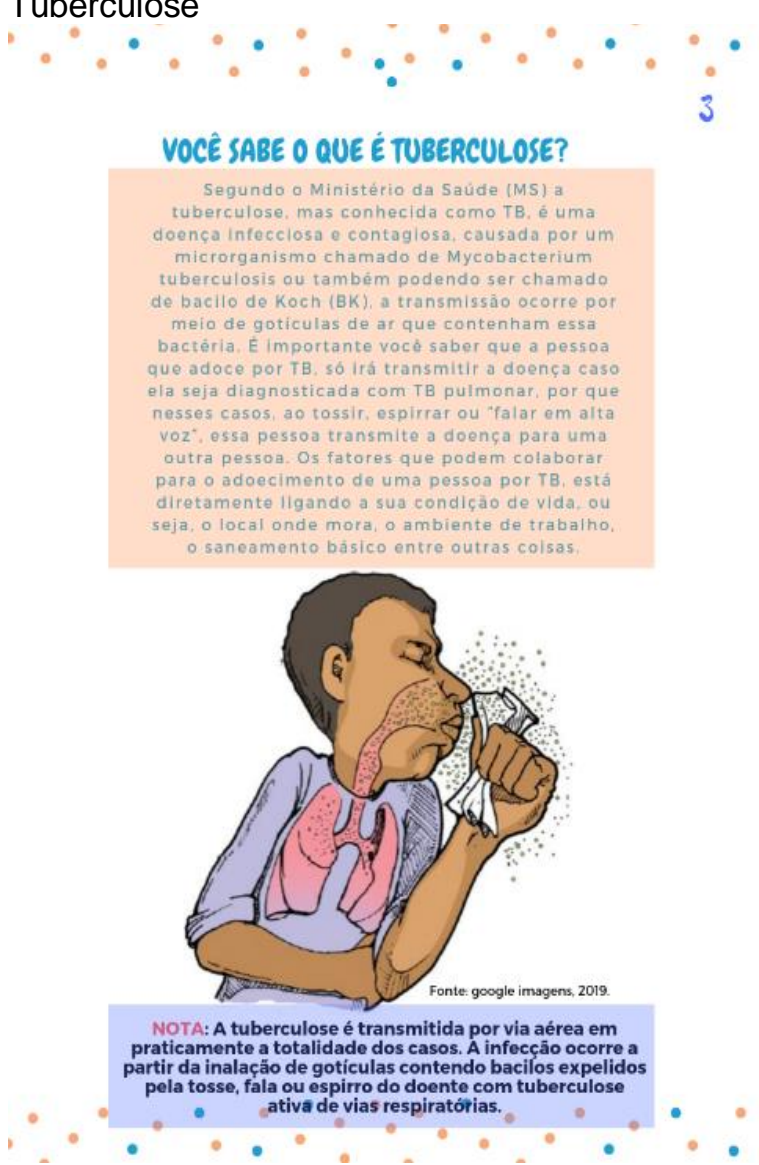

Fonte: acervo pessoal dos autores 
Figura 5: Informações adicionais sobre 0 tratamento de Tuberculose

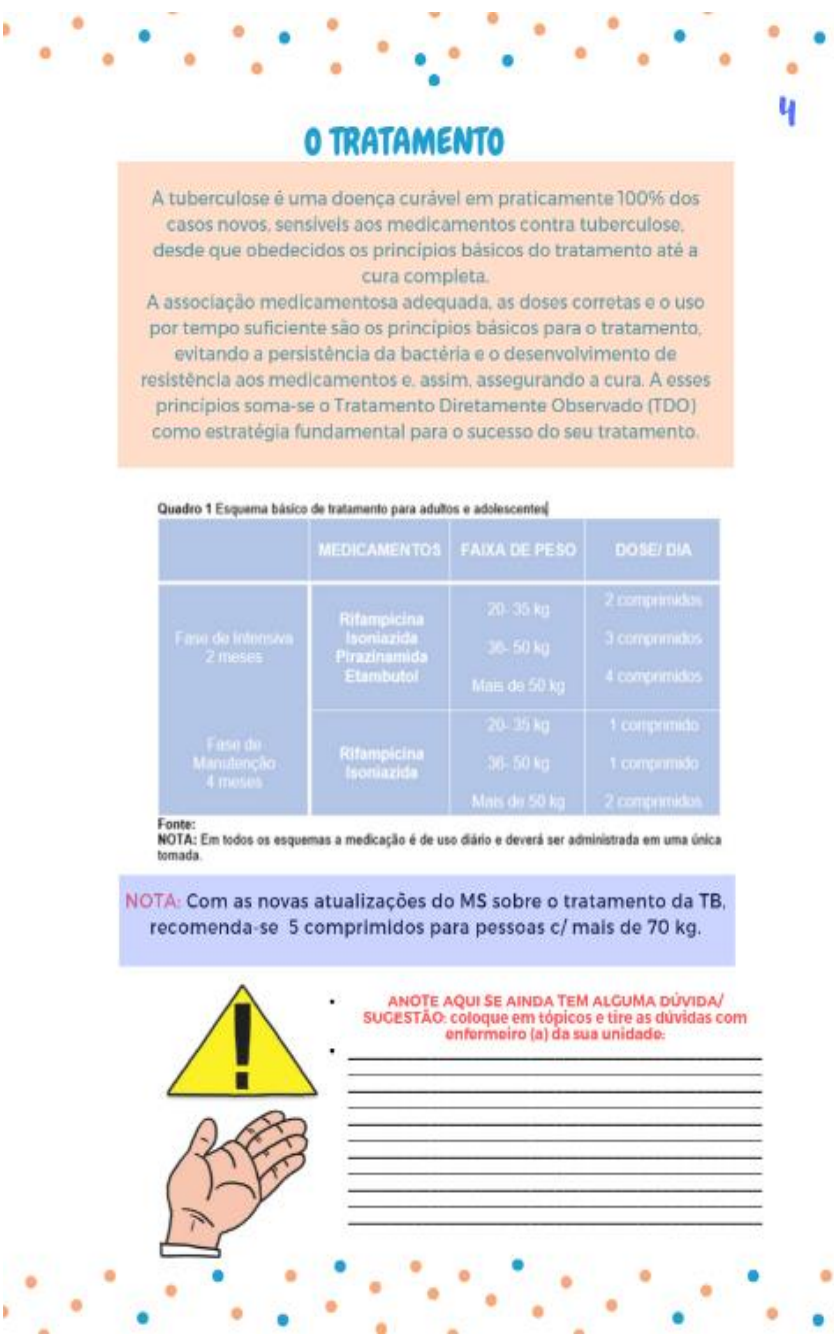

Fonte: acervo pessoal dos autores
Figura 6: Informações adicionais sobre o TDO

\section{O QUE É O TRATAMENTO DIRETAMENTE OBSERVADO?}

Voce sabia que existo um tratamento diferenciado para Tuberculose?

Um tratamento pactuado com o Ministério da Saúde e pensando nos usuários com Tuberculose?

Um tratamento que pode ser acompanhado dia-a-dia pelo profissional de saúde do seu território e que vocé pode contar com ele todos os dias?

Esse tratamento é conhecido como TODO, ou TRATAMENTO

DIRETAMENTE OBSERVADO, essa estratégia consiste na observação da tomada do medicamento pelo profissional de saúde, de preferéncia, todos os dias (de segunda a sexta). nos dois primeiros meses (Fase de Ataque) e no minimo trés vezes por semana nos ùltimos quatro meses (Fase de Manutençăo). Entretanto nos finais de semana e feriad05, os medicamentos são distribuidos aos usuários para as doses autoadministradas

orlentados adequadamente para fazer a tomada do

medicamento corretamente. Essa estratégia tem grandes. indices de positividade na eliminaçâa da Tuberculose e de suas formas mais graves e resistentes (TB-MR)

Essa estratégia alèm de benéfica para o usuário auxilia o

profissional no controle da doença no território adstrito da unidade basica ou estrategla saúde da familia, mas tambem contribui para tirada de duvidas do usuário, complementaçâo de informaçōes e no vinculo.

O culdado é a nossa maior virtude e a nossa assistência ê melhor ataque para a eliminaçà dessa doenca do nosso pais. entâo venha nos ajudar, venha participar da estratégia TDO e contribua para sua melhora e pra o nosso serviço.

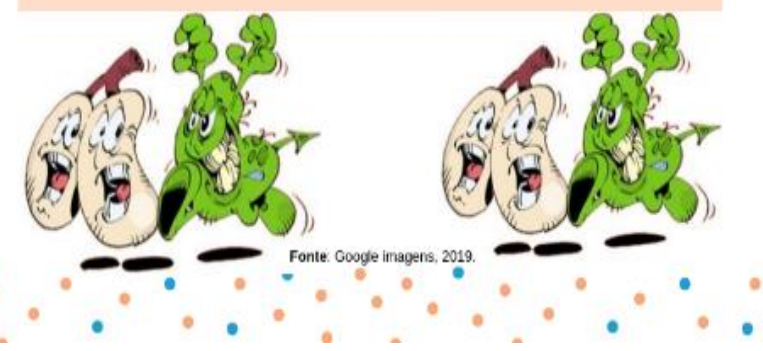

Fonte: acervo pessoal dos autores 
Figura 7: Informações adicionais sobre os efeitos adversos do tratamento de Tuberculose

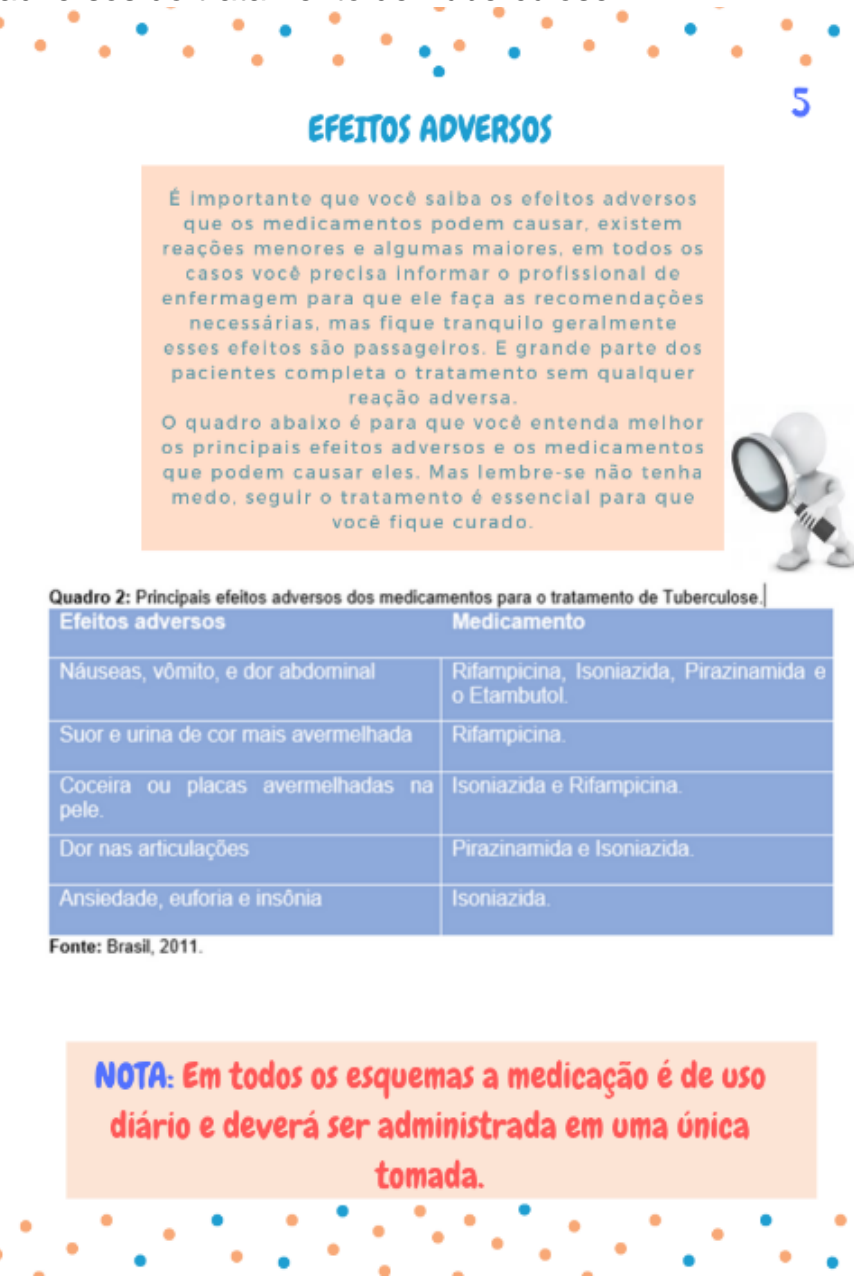

Fonte: acervo pessoal dos autores
Figura 8: conteúdo interno do Planner contendo aprazamento pessoal do usuário

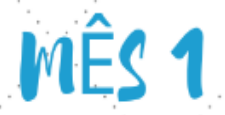

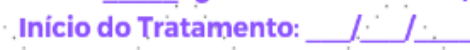
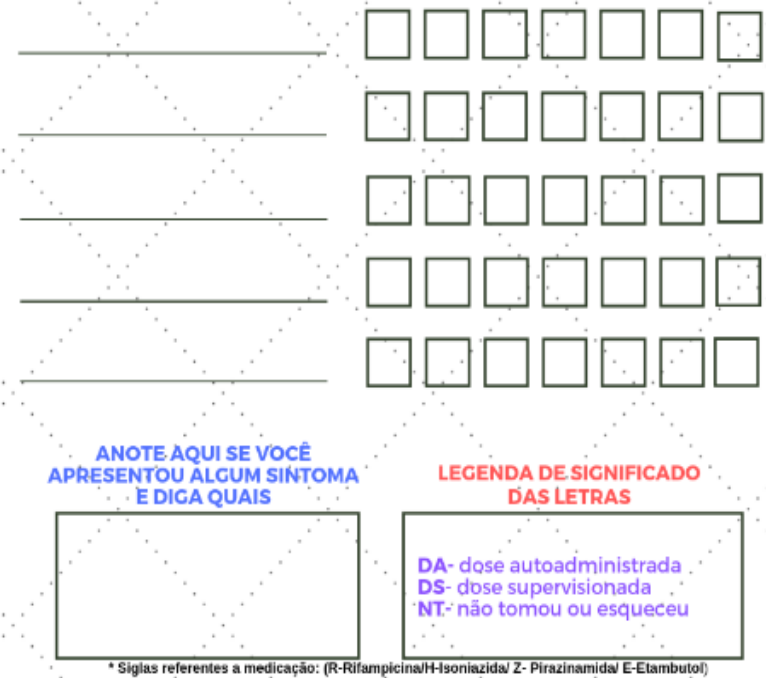

Fonte: acervo pessoal dos autores 


\section{Como citar este artigo}

Amorim CSS; Oliveira FC; Barbosa VTP; Costa MCP; Santos SFD; Silva MPSDC. Produção de um planner para assistência de enfermagem no tratamento diretamente observado. Revista de Saúde Digital e Tecnologias Educacionais. [online], volume 4, n. 2. Editor responsável: Luiz Roberto de Oliveira. Fortaleza, mês e ano, p. 13-25. Disponível em: http://periodicos.ufc.br/resdite/index. Acesso em "dia/mês/ano".

Data de recebimento do artigo: 08/08/2019

Data de aprovação do artigo: 19/11/2019

RE. SAÚD. DIGI. TEC. EDU., Fortaleza, CE, v.4, n.2, p.13-25, ago./dez. 2019. 